\title{
A Dual-mesh Framework for Multiphysics Simulation of Photoconductive Terahertz Devices
}

\author{
Liang Chen* and Hakan Bagci \\ Division of Computer, Electrical, and Mathematical Science and Engineering, \\ King Abdullah University of Science and Technology (KAUST), \\ Thuwal 23955-6900, Saudi Arabia \\ \{liang.chen, hakan.bagci\}@kaust.edu.sa
}

\begin{abstract}
The operation of a photoconductive terahertz (THz) device relies on optoelectronic interactions and $\mathrm{THz}$ radiation. Although these two processes are coupled, in simulations, they are often modeled separately due to the large difference between the frequencies of optical and $\mathrm{THz}$ electromagnetic waves. To model both processes in a single simulation, we propose a dual-mesh discontinuous Galerkin (DG) scheme. Optoelectronic interactions and $\mathrm{THz}$ radiation are accounted for by solving, respectively, a coupled system of Maxwell and drift-diffusion equations discretized on a fine mesh and only the Maxwell equations discretized on a coarse mesh. This approach uses an efficient high-order interpolation scheme to "connect" electric field and current discretized on these two meshes. The proposed scheme is validated against experimental results.
\end{abstract}

\section{Introduction}

Terahertz (THz) technologies have numerous applications ranging from wireless communication to nondestructive imaging. One of the main challenges in realizing these technologies is to design and fabricate efficient, compact, and frequency-stable $\mathrm{THz}$ sources that can operate at room temperatures. Photoconductive (PC) $\mathrm{THz}$ devices, including $\mathrm{PC}$ antennas (PCAs) and photomixers (PMs), are promising candidates in fulfilling this challenge [1,2]. In recent years, the performance of $\mathrm{PC} \mathrm{THz}$ devices has improved significantly with the inclusion of metallic plasmonic nanostructures and dielectric metasurfaces in their design $[1,2]$. To realize these designs, one needs to use simulation tools that can accurately account for nonlinear interactions between electromagnetic (EM) waves and carrier dynamics and model the geometrically intricate nanostructures. We should also note here that the simulations should be as fast as possible, since an efficient simulation tool can significantly speed-up the design cycle and reduce its cost.

The operation of a $\mathrm{PC} \mathrm{THz}$ device relies on interactions between optical and THz EM waves, with wavelengths of $800 \mathrm{~nm}$ and $0.3 \mathrm{~mm}$, respectively. Moreover, the optical and $\mathrm{THz} \mathrm{EM}$ waves are coupled through the dynamic carrier response in the semiconductor layer, which introduces another length scale of $10 \mathrm{~nm}$. The corresponding time scales of the dynamics of optical EM wave, $\mathrm{THz}$ EM wave, and carriers are $1 \mathrm{fs}, 1 \mathrm{ps}$, and $0.1 \mathrm{ps,}$ respectively. These wide ranges of length and time scales make the simulations of the $\mathrm{PC} \mathrm{THz}$ devices very challenging. Currently, simulations focus on either the optoelectronic interactions (interactions of optical EM wave and carriers) in the nanostructured semiconductor layer, or the $\mathrm{THz}$ radiation where the optoelectronic component of the device is only effectively considered as a source port of the $\mathrm{THz}$ antenna [3].

However, the current produced by the optoelectronic component has a non-uniform profile in space and this profile changes with the impedance of the THz antenna [4]. Therefore, the overall response of a $\mathrm{PC} \mathrm{THz}$ device depends on the design of both the optoelectronic component and the $\mathrm{THz}$ antenna as well as the two-way feedback between them. Therefore, numerical tools that can model the optoelectronic component, the THz antenna, and their coupling in a single simulation are required. However, accommodating a $\mathrm{THz}$ antenna (of size $\sim 1 \mathrm{~mm}$ ) within the simulation of the optoelectronic component is computationally too expensive, since the mesh size has to be selected according to the optical wavelength $(\sim 800 \mathrm{~nm})$.

In this work, a dual-mesh framework is proposed for simultaneously modeling the optoelectronic interactions and the $\mathrm{THz}$ radiation on $\mathrm{PC} \mathrm{THz}$ devices. A discontinuous Galerkin (DG) scheme is used to solve the coupled system of time-dependent Maxwell and drift-diffusion (DD) equations on a fine mesh discretizing the semiconductor layer. The nonlinear coupling between the optical EM wave and the carriers are fully considered. The $\mathrm{THz}$ radiation from the antenna is computed using a DG scheme that solves only Maxwell equations on a course mesh since the high frequency components of the photocurrent can be ignored. Thanks to the high flexibility of meshing and the high-order accuracy that comes with both of the DG schemes, the solutions on the two different scale meshes are efficiently "connected" using a high-order interpolation algorithm on the fly during time marching. Using two different meshes for high-frequency (optical) and the lowfrequency $(\mathrm{THz})$ simulations results in a computationally efficient framework that makes the direct simulation of PC $\mathrm{THz}$ devices feasible. 


\section{Formulation}

\section{A. Optoelectronic Interactions}

The operation of a PC THz device has two physical stages: a nonequilibrium steady-state under a static bias voltage and a transient stage that starts when the optical excitation enters the device as a pump. The steady-state is described by a coupled system of Poisson and stationary DD equations, which is solved by a (nonlinear) DG solver we developed previously [5]. The transient stage is described by a coupled system of time-dependent Maxwell and DD equations. This system takes into account the nonlinear coupling between EM waves and carrier dynamics. In this work, we focus on the transient stage and tackle the challenge of modeling the optoelectronic response and the $\mathrm{THz}$ radiation in a single simulation. The Maxwell-DD system reads [3]

$$
\begin{gathered}
\mu(\mathbf{r}) \partial_{t} \mathbf{H}^{t}(\mathbf{r}, t)=-\nabla \times \mathbf{E}^{t}(\mathbf{r}, t) \\
\varepsilon(\mathbf{r}) \partial_{t} \mathbf{E}^{t}(\mathbf{r}, t)=\nabla \times \mathbf{H}^{t}(\mathbf{r}, t)-\left[\mathbf{J}_{e}^{t}(\mathbf{r}, t)+\mathbf{J}_{h}^{t}(\mathbf{r}, t)\right] \\
q \partial_{t} n_{c}^{t}(\mathbf{r}, t)= \pm \nabla \cdot \mathbf{J}_{c}^{t}(\mathbf{r}, t)-q\left[R^{t}\left(n_{e}^{t}, n_{h}^{t}\right)-G\left(\mathbf{E}^{t}, \mathbf{H}^{t}\right)\right] \\
\mathbf{J}_{c}^{t}(\mathbf{r}, t)= \pm q V_{T} \mu_{c}\left(\mathbf{E}^{s}\right) \nabla n_{c}^{t}(\mathbf{r}, t)+q \mu_{c}\left(\mathbf{E}^{s}\right) \\
\left(\mathbf{E}^{s}(\mathbf{r}) n_{c}^{t}(\mathbf{r}, t)+\mathbf{E}^{t}(\mathbf{r}, t)\left[n_{c}^{t}(\mathbf{r}, t)+n_{c}^{s}(\mathbf{r}, t)\right]\right)
\end{gathered}
$$

where the upper and lower signs should be selected for electrons $(c=e)$ and holes $(c=h)$, respectively. Here, $\mathbf{E}^{t}(\mathbf{r}, t)$ and $\mathbf{H}^{t}(\mathbf{r}, t)$ are the EM fields, $n_{c}^{t}(\mathbf{r}, t), c \in\{e, h\}$ are the carrier densities, $\mathbf{J}_{c}^{t}(\mathbf{r}, t)$ the carrier current, $R^{t}\left(n_{e}^{t}, n_{h}^{t}\right)$ and $G\left(\mathbf{E}^{t}, \mathbf{H}^{t}\right)$ are the recombination and carrier generation rates [3], $\mathbf{E}^{s}$ is the static electric field resulting from the bias voltage, $\mu_{c}\left(\mathbf{E}^{s}\right)$ is the fielddependent mobility, $\varepsilon(\mathbf{r})$ and $\mu(\mathbf{r})$ are the permittivity and permeability, and $V_{T}$ is the thermal voltage. The term $\mathbf{E}^{t}(\mathbf{r}, t)\left[n_{c}^{s}(\mathbf{r}, t)+n_{c}^{t}(\mathbf{r}, t)\right]$ in Equation (4) represents the drift motion of the carriers driven by the optical EM wave and its contribution to the current is ignored since $\left|\mathbf{E}^{s}(\mathbf{r}, t)\right| \gg\left|\mathbf{E}^{t}(\mathbf{r}, t)\right|$ and the oscillation period of $\left|\mathbf{E}^{t}(\mathbf{r}, t)\right|$ is much smaller than the drift time of carriers.

Equations (1)-(4) are discretized using the time-domain DG scheme [3]. For Maxwell equations, the upwind flux is used. For DD equations, the LDG alternate flux is used for the diffusion term and the local Lax-Friedrichs flux is used for the drift term. The resulting matrix systems are integrated in time to obtain the unknowns $\mathbf{E}^{t}(\mathbf{r}, t)$, $\mathbf{H}^{t}(\mathbf{r}, t)$, and $n_{c}^{t}(\mathbf{r}, t), c \in\{e, h\}$ using the explicit fourthorder five-stage low-storage and the third-order total variation diminishing Runge-Kutta schemes, respectively. The solutions of the matrix systems are fed into each other in an alternating fashion through the coupling terms.

\section{B. The Dual-Mesh Scheme}

The DG scheme described above is used to model the optoelectronic interactions only in the vicinity of the nanostructured semiconductor layer. In principle, since this
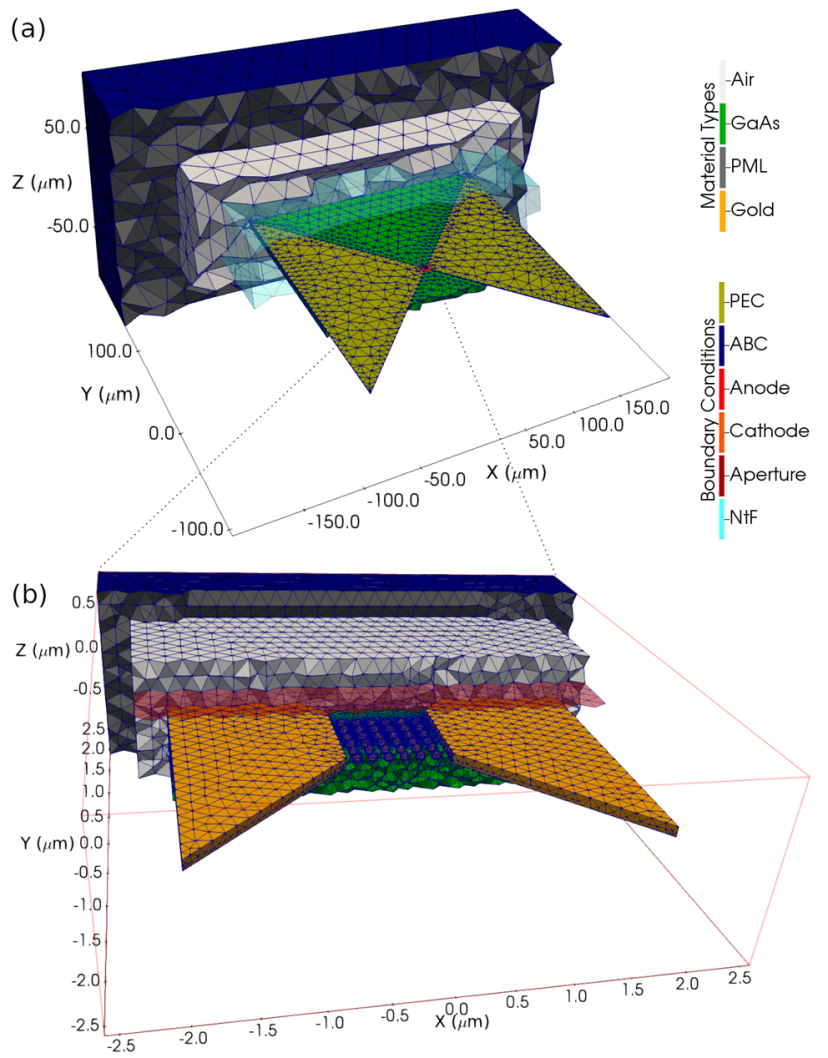

Figure 1. Simulation set up. (a) Coarse mesh discretizing the $\mathrm{THz}$ antenna and (b) fine mesh discretizing the optoelectronic component.

scheme solves Maxwell equations, it can also account for the $\mathrm{THz}$ antenna. However, discretizing the antenna (of size $\sim 1 \mathrm{~mm}$ ) with the mesh element size ( $20 \sim 80 \mathrm{~nm}$ ) required by the optoelectronic interactions would result in $\sim 10^{13}$ mesh elements.

Therefore, the $\mathrm{THz}$ antenna is discretized using a separate coarse mesh and accounted for using a DG scheme that solves only the Maxwell equations. Hereafter, we denote the mesh (and corresponding solver) used for the $\mathrm{THz}$ antenna and the optoelectronic component with $\mathrm{A}$ and $\mathrm{B}$, respectively. See Figures 1(a) and (b) for examples of meshes A and B. Mesh B is truncated with a box bounding the semiconductor region and the metal contacts of the $\mathrm{THz}$ antenna. The current density on the metal contacts obtained by solver $\mathrm{B}$ is used to excite the $\mathrm{THz}$ antenna modeled by solver A. The THz EM fields obtained by solver A are coupled back to solver $\mathrm{B}$ in the overlapping region of the meshes. The exchange of data between solvers is handled on-the-fly during time marching using an interpolation algorithm as described next.

Let $f\left(\mathbf{r}_{n}\right)$ represent the solutions computed at nodal points $\mathbf{r}_{n}, n=1, \ldots, N_{p}$, within element $K$. Then, the interpolated $f\left(\mathbf{r}_{i}^{I}\right)$ at a set of target points $\mathbf{r}_{i}^{I}, i=1, \ldots, M$, are obtained using

$$
\bar{f}_{I}=\bar{P} \bar{f}
$$




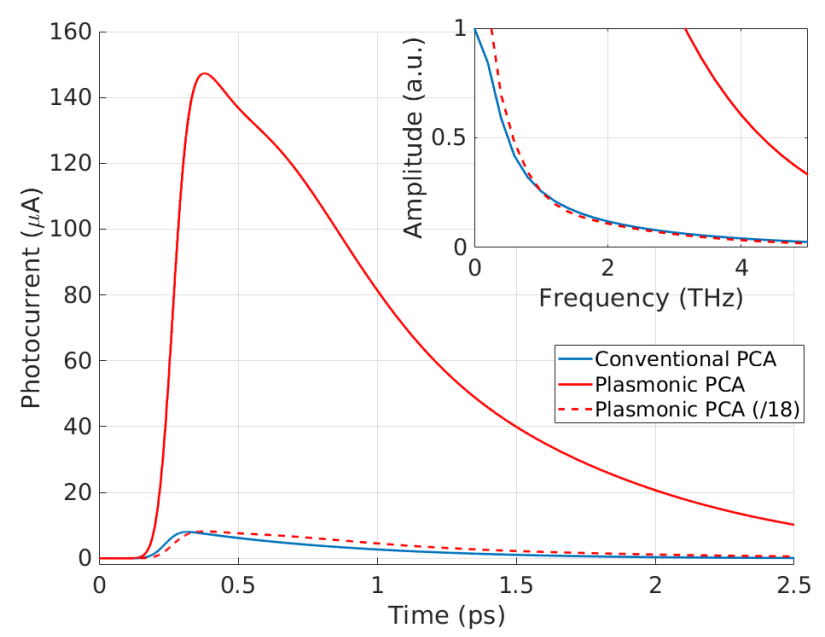

Figure 2. Photocurrents and corrsponding power spectra for conventional and plasmonic PCAs.

Here, $\bar{f}_{I}=\left[f\left(\mathbf{r}_{1}^{I}\right), \ldots, f\left(\mathbf{r}_{M}^{I}\right)\right]^{T}, \quad \bar{f}=\left[f\left(\mathbf{r}_{1}\right), \ldots, f\left(\mathbf{r}_{N_{n}}\right)\right]^{T}$, $\bar{P}=\bar{V}_{I} \bar{V}^{-1}$ is the interpolation matrix, $\bar{V}_{I}$ and $\bar{V}$ are generalized Vandemonde matrices with entries $\bar{V}_{I}(i, j)=\phi_{j}\left(\mathbf{r}_{i}^{I}\right)$ and $\bar{V}(i, j)=\phi_{j}\left(\mathbf{r}_{i}\right)$, and $\phi_{j}(\mathbf{r})$ is the $j$-th orthonormal polynomial basis [6]. Here, $\mathbf{r}_{n}$ and $\mathbf{r}_{i}^{I}$ are coordinates in the reference element and are obtained using the mapping $\mathbf{r}=\psi(\mathbf{R})$ where $\mathbf{R}$ is the physical coordinate. The interpolation operation is only required on a limited number of elements. For example, when transferring $\mathbf{J}(\mathbf{r})$ from solver A to solver B, since mesh B is significantly coarser than mesh $\mathrm{A}$, only a small part of the elements in mesh A contain $\mathbf{r}_{i}^{I}$. Conversely, when transferring $\mathbf{E}(\mathbf{r})$ from solver B to solver A, only a small number of source points $\mathbf{r}_{n}$ is used. The local interpolation matrices are pre-calculated and re-used during the explicit time iterations.

\section{Numerical Examples}

To validate the proposed scheme, we simulate a plasmonic enhanced $\mathrm{THz}$ PCA. Figure 1 describes the simulation setup with (a) and (b) showing the coarse and fine meshes. The flare angle, gap width and arm length of the $\mathrm{THz}$ bowtie antenna are $90^{\circ}, 1 \mu \mathrm{m}$ and $100 \mu \mathrm{m}$, respectively. For the nanostructures, the repetition period and the diameter of the sphere are $0.15 \mu \mathrm{m}$ and $0.1 \mu \mathrm{m}$, respectively. The material parameters are same as those in $[3,7]$. Elements sizes in meshes $\mathrm{A}$ and $\mathrm{B}$ change in ranges $[2,60] \mu \mathrm{m}$ and $[0.02,0.1] \mu \mathrm{m}$ resulting in 112,250 and 370,286 elements, respectively.

The aperture source injects a laser pulse from top to bottom. The pulse shape is same as that in [3,7]. Figure 2 shows the generated photocurrent on the electrodes. The signal pulse shape and its power spectrum match with those of the conventional PCA (without nanostructures), with an enhancement factor of approximately 18 . The decay time

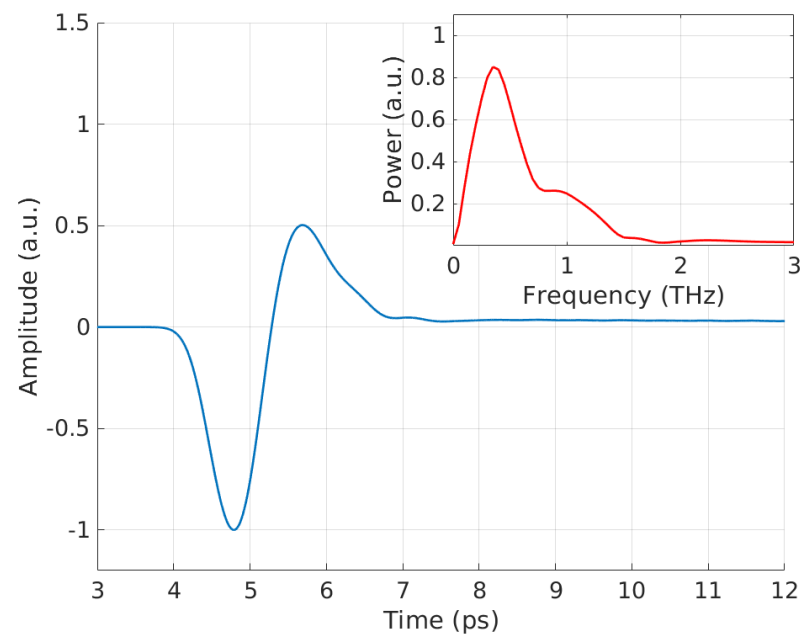

Figure 3. Detected $\mathrm{THz}$ signal and corresponding power spectrum.

is mainly determined by the carrier life time. Those results agree with the properties of typical PCAs observed in experiments $[1-3,7]$.

The THz radiation signal is obtained by applying the time domain near-to-far field transformation to the EM fields on the "NtF" box shown in Figure 1 (a). Assuming that the same PCA is used for detection, the received signal is obtained following the method in [8,9]. Figure 3 shows the detected $\mathrm{THz}$ signal and its power spectrum, which qualitatively agree with those obtained with similar bowtie $\mathrm{THz}$ antennas [10,11]. The radiation spectrum and efficiency of $\mathrm{THz}$ PCAs critically depend on the characteristics of the photocurrent and the geometry of the $\mathrm{THz}$ antenna [4]. In future works, the proposed framework will be used for parametric studies of different types of $\mathrm{THz}$ antennas, such as H-type dipole, strip line, and logarithmic spiral antenna $[1,2,10]$.

\section{Acknowledgements}

This publication is supported by the King Abdullah University of Science and Technology (KAUST) Office of Sponsored Research (OSR) under Award No 2016-CRG52953. The authors would like to thank the King Abdullah University of Science and Technology Supercomputing Laboratory (KSL) for providing the required computational resources.

\section{References}

1. N. T. Yardimci and M. Jarrahi, "Nanostructure Enhanced Photoconductive Terahertz Emission and Detection," Small, vol. 14, no. 44, pp. 1802437, 2018.

2. S. Lepeshov, A. Gorodetsky, A. Krasnok, E. Rafailov, and P. Belov, "Enhancement of terahertz photoconductive antenna operation by optical nanoantennas," Laser Photon. Rev., vol. 11, no. 1, pp. 1600199, 2017. 
3. L. Chen and H. Bagci, "Multiphysics Modeling of Plasmonic Photoconductive Devices using Discontinuous Galerkin Methods," arXiv: 1912.03639, 2019.

4. F. Miyamaru, Y. Saito, K. Yamamoto, T. Furuya, S. Nishizawa and M. Tani, "Dependence of emission of terahertz radiation on geometrical parameters of dipole photoconductive antennas," App. Phys. Lett., vol. 96, no. 21, pp. 211104, 2010.

5. L. Chen and H. Bagci, "Steady-state Simulation of Semiconductor Devices using Discontinuous Galerkin Methods," IEEE Access, pp. 1-1, 2020, doi: 10.1109/ACCESS.2020.2967125.

6. J. Hesthaven and T. Warburton, Nodal Discontinuous Galerkin Methods: Algorithms, Analysis, and Applications. NY, USA: Springer, 2008.

7. E. Moreno, M. F. Pantoja, S. G. Garcia, A. R. Bretones, and R. G. Martin, "Time-domain numerical modeling of THz photoconductive antennas," IEEE Trans. Terahertz Sci. Tech., vol. 4, no. 4, pp. 490-500, 2014.

8. P. U. Jepsen, R. H. Jacobsen, and S. R. Keiding, "Generation and detection of terahertz pulses from biased semiconductor antennas," J. Opt. Soc. Am. B, vol. 13, no. 11, pp.2424-2436, 1996.

9. M. Tani, Y. Hirota, C. T. Que, S. Tanaka, R. Hattori, M. Yamaguchi, S. Nishizawa, and M. Hangyo, "Novel terahertz photoconductive antennas," Int. J. Infrared Millimeter Waves, vol. 27, no. 4, pp. 531-546, 2006.

10. M. Tani, S. Matsuura, K. Sakai, and S. Nakashima, "Emission characteristics of photoconductive antennas based on low-temperature-grown GaAs and semiinsulating GaAs," Appl. Opt., 36, pp. 7853-7859, 1997.

11. S.-G. Park, Y. Choi, Y. J. Oh, and K. H. Jeong, "Terahertz photoconductive antenna with metal nanoislands," Opt. Express, vol. 20, no. 23, pp. 2553025535, 2012. 